\title{
Diagnóstico de la Situación de las Mipymes del Sector Agropecuario en Yucatán
}

\section{Diagnosis of the Situation of MSMEs in the Agricultural Sector in Yucatan}

\author{
Moo Novelo Carlos Antonio*, Hernández Cuevas Francisco Iván**, \\ Mex Arriaga Fredi Israel ${ }^{\star \star *}$
}

\begin{abstract}
*Maestro en Gobierno y Políticas Públicas, profesor de tiempo completo. Universidad Tecnológica Metropolitana. Email: carlos.moo@utmetropolitana.edu.mx, ORCID: https://orcid.org/0000-0002-05381944.

**Doctor en Ciencias Sociales, Profesor de Asignatura. Universidad Marista de Mérida. Email: franciscohernandezc@p.marista.edu.mx, ORCID: https://orcid.org/0000-0002-1310-7574.

${ }^{* * *}$ Maestro en Planificación de Empresas y Desarrollo Regional, Profesor de Asignatura. Universidad Tecnológica Metropolitana. Email: fredi.mx@pa.metropolitana.edu.mx, ORCID: https://orcid.org/00000002- 5727-3347.
\end{abstract}

Correo para recibir correspondencia: carlos.moo@utmetropolitana.edu.mx

Fecha de recibido: 5 de noviembre de 2020

Fecha de aceptación: 25 de mayo de 2021

MOO-NOVELO, C. A., HERNÁNDEZ-CUEVAS, F. I., MEX-ARRIAGA, F. I.

HITOS DE CIENCIAS ECONÓMICO ADMINISTRATIVAS SEPTIEMBRE-DICIEMBRE 2021. Año 27, No. 79. Págs. 310 -328 310 


\title{
RESUMEN
}

El artículo muestra las deficiencias administrativas y organizacionales que presentan las MIPYMES ubicadas en el sector agropecuario del estado de Yucatán, así como también las principales variables que inciden en su posibilidad de crecimiento económico.

OBJETIVO: Identificar la situación administrativa que predomina en las microempresas agropecuarias en nueve municipios del estado de Yucatán, y que permita determinar los principales problemas que se presentan en su administración y organización.

MATERIAL Y MÉTODO: En este caso en particular se realizó la selección de las empresas a través del muestreo no aleatorio y por conveniencia en diversas partes del estado de Yucatán, debido a que se tuvo contacto previo con los dueños de las empresas a los cuales se les aplicó el cuestionario de los municipios donde se realizó el estudio.

RESULTADOS: De los principales resultados obtenidos destaca que el 77\% de las empresas son de tamaño micro y el $23 \%$ son pequeñas, además el $55.5 \%$ de las empresas tienen una estructura orgánica establecida, pero no la siguen, mientras que el $44.5 \%$ de ella dijo no tener definido una estructura orgánica para su empresa.

CONCLUSIONES: Es evidente que la carencia de una estructura organizativa bien establecida genera dificultades a las MIPYMES, para poder potencializar sus actividades, ya que, al evitar el ciclo administrativo en estas empresas, carecen de rumbo y tienen bien planificado hasta donde quieren llegar.

PALABRAS CLAVE: Diagnóstico. Estructura administrativa. Sector Agropecuario.

\begin{abstract}
The article shows the administrative and organizational deficiencies presented by the MSMEs located in the agricultural sector of the state of Yucatán, as well as the main variables that affect their possibility of economic growth.
\end{abstract}


DIAGNÓSTICO DE LA SITUACIÓN DE LAS MIPYMES DEL SECTOR AGROPECUARIO EN YUCATÁN.

OBJECTIVE: To identify the administrative situation that predominates in agricultural microenterprises in nine municipalities of the state of Yucatan, and that this action allows to determine the main problems that arise in their administration, and organization.

MATERIAL AND METHOD: In this case, the companies were selected through non-probability sampling belonging to various parts of the state of Yucatán, due to prior contact with the owners of those companies, the questionnaire was applied to them, in the municipalities where the study was carried out.

RESULTS: From the results obtained, $77 \%$ are micro enterprises, $23 \%$ are small, $55.5 \%$ have an established organic structure although they do not follow it, meanwhile a $44.5 \%$ does not have a defined organic structure in their enterprises.

CONCLUSIONS: It is evident that the lack of a well-established organizational structure creates difficulties for MSMEs, to be able to potentiate their activities, by avoiding the administrative cycle in these companies, they lack direction and have well planned where they want to go.

KEY WORDS: Diagnosis. Administrative Structure. Agricultural Sector.

\section{INTRODUCCIÓN}

Las micro, pequeñas y medianas empresas (MIPYMES) tienen una gran relevancia para la economía de los países en desarrollo ya que contribuyen con la generación de los empleos que se requieren, por lo mismo estas empresas pueden ser del sector servicios, industrial y agropecuario. Góngora (2013) menciona que en casi todos los países el 90\% de las empresas son MIPYMES, estas contribuyen en buena medida a la generación de empleos en las zonas en donde se desarrollan. En el caso de México de acuerdo con la Encuesta Nacional sobre Productividad y Competitividad de las Micro, Pequeñas y Medianas empresas (ENAPROCE 2015) el $97.62 \%$ de todas las empresas que operan en el país son microempresas, misma situación en el sector agropecuario en donde el $81.48 \%$ de las empresas perteneces a este tamaño (García, 2017), de ahí su importancia por identificar el cómo se administran y qué 
DIAGNÓSTICO DE LA SITUACIÓN DE LAS MIPYMES DEL SECTOR AGROPECUARIO EN YUCATÁN.

dificultades presentan, además que como se ha mencionado son parte importante de toda la economía.

De acuerdo con datos del INEGI (2018) se destaca que de 100 negocios que entran al mercado, tan solo en el primer año desaparecen el 33\%, mientras que a la mortandad acumulada en el quinto año llega al $65 \%$ de cada 100 empresas, razón por la cuál es importante identificar el cómo contribuye la organización administrativa a reducir estos números más en sector en donde existe muchas más implicaciones por la naturaleza de la actividad.

En este sentido, la ley para el Desarrollo de la Competitividad de la Micro, Pequeña y Mediana Empresa (DOF 2017), se menciona la clasificación de las empresas de acuerdo con el número de empleados y actividad que desarrolla.

\section{Tabla 1}

Clasificación de las empresas según tamaño

\begin{tabular}{cccc}
\hline \multicolumn{4}{c}{ Estratificación por número de trabajadores } \\
\hline Sector/ tamaño & Industria & Comercio & Servicios \\
\hline Micro & $0-10$ & 0 a 10 & 0 a 10 \\
Pequeña & 11 a 50 & 11 a 30 & 11 a 50 \\
Mediana & 51 a 250 & 31 a 100 & 51 a 100 \\
\hline
\end{tabular}

Fuente: Elaboración propia con datos de la Ley para el Desarrollo de la Competitividad de la Micro, Pequeña y Mediana Empresa (2017).

En México, la mayoría de las empresas de todos los sectores tiene en promedio de 1 a 10 trabajadores, lo mismo sería para las actividades agropecuarias. En el caso de Yucatán, de acuerdo con el Directorio Estadístico Nacional de Unidades Económicas - DENUE (2019) las empresas del sector agropecuario representan un total de 1,268 en todo el estado, donde el $64.35 \%$ (816) tienen de 0 a 5 empleados, el 15.61\% (198) de 6 a 10 empleados, el 10.96\% (139) tienen de 11 a 30 trabajadores, el 3.78\% (48) tienen de 31 a 50 empleados, mientras que el $2.76 \%$ (35) emplea de 51 a 100 personas, el 1.73\% (22) en estas se encuentran ocupadas de 101 a 250 personas y en el $0.78 \%$ (10) ocupan de 251 y más trabajadores.

El sector agropecuario para las economías nacionales tiene una gran importancia debido a que su actividad es la encargada de proveer de los recursos necesarios para la alimentación de los individuos que conforman la sociedad, al mismo tiempo, proporciona las materias primas para que el sector industrial transforme y pueda de esa manera crecer y contribuir al Producto 

Interno Bruto (PIB). De ahí, que la importancia radica en implementar mecanismos que permitan generar oportunidades de desarrollo para las empresas del sector agropecuario. Por otro lado se encuentran las empresas formales de producción agropecuaria en donde en su mayoría predomina una administración simple, ya que la mayoría de las decisiones son tomadas por el dueño, quien funge como gerente, encargado de compras y vendedor, razón por la cual es importante conocer si esta forma de administración influye o no en su proceso de crecimiento y consolidación de estas empresas o si lo que necesitan es mayor financiamiento o no, para consolidarse, pero para tener claridad en lo que requieren es importante conocer cuál es su situación organizativa; es decir, cómo se administra y se toman las decisiones.

Las microempresas, son importantes para el desarrollo económico de una zona, región o país, ya que son fuentes generadoras de trabajo, este tipo de empresas puede surgir en cualquiera de los sectores económico en las que se divide el país. Sin embargo, una empresa agropecuaria es de vital importancia no solo porque genera los empleos en las zonas rurales, sino que también ofrece los insumos y la materia prima que el sector industrial necesitará para generar su proceso de producción.

De acuerdo con el Censo Económico 2019 realizado por el INEGI, los resultados preliminares mencionan un total de cuatro millones 773 mil 995 unidades económicas, de estas se dividen en sectores de actividad económica como las manufacturas con 12.19\% (582 mil 013 unidades), el comercio representa el grueso de las unidades económicas de México con el 46.64\% (2 millones 227 mil 058 unidades), el rubro de los servicios privados no financieros está integrado por el 39.14\% (millón 868 mil 993 empresas) y dentro de la clasificación resto de las actividades se consideraron al 2.00\% (95 mil 931 empresas), es en este rubro en donde se puede encontrar a las organizaciones que realizan alguna actividad referente al sector agropecuario.

En ese total de las unidades económicas emplean a 26 millones 561 mil 457 personas, que en el caso de la actividad manufacturera le da empleos al seis millones 555 mil 790 personas, en las actividades de comercio se emplean a 7 millones 429 mil 763 personas, por el contrario en el sector servicios privados no financieros se emplean a 9 millones 542 mil 420 personas, y en el rubro de resto de actividades ahí se emplean a 3 millones 33 mil 484 personas, que precisamente son los que desarrollan las actividades propias del sector agropecuario. 
DIAGNÓSTICO DE LA SITUACIÓN DE LAS MIPYMES DEL SECTOR AGROPECUARIO EN YUCATÁN.

De manera específica, en el resto de las actividades económicas se encuentra clasificada las actividades referentes a: pesca, avicultura y servicios relacionados con las actividades agropecuarias que está conformada por 24 mil 326 unidades económicas y que representa el $25.35 \%$ del total de ese rubro. En el caso específico de Yucatán en la clasificación catalogada como resto de las actividades económicas se tiene un total de 3,172 unidades y representa el $2.86 \%$ del total estatal, de las cuales 1,267 de las empresas realizan actividades vinculadas con el sector agropecuario.

Del mismo modo, datos del Censo económico 2019, muestran que del total de las unidades económicas que operan en México, el 95\% son de tamaño micro, en donde se emplea el 37.8\% de las personas; las pequeña empresas representan el $3.95 \%$ y dan empleo a $15.9 \%$ de la personas, en tanto que las empresas de tamaño mediano, emplean al $15.9 \%$ de la personas y del total de las unidades únicamente representan al $0.83 \%$, mientras que las empresas grandes representan del total el $0.23 \%$ pero generan el $31.6 \%$ de los empleos.

Ante esto, es de suponer que las unidades económicas agropecuarias considerados en la clasificación denominado resto de las actividades económicas con un total de 95,031, unidades económicas a nivel nacional, se integren de la siguiente manera el 69.19\% de esas empresa en el área agropecuaria son de tamaño micro, el $22.82 \%$ son pequeñas unidades productoras, y el $6.5 \%$ están clasificados como medianas empresas, y solamente el $1.46 \%$ son grandes empresas que realizan una función propia de empresas, estas en la mayoría de los casos son trasnacionales.

De acuerdo con la Encuesta Nacional Agropecuaria 2017, Algunos de los principales problemas que limitan el crecimiento de las empresas agropecuarias son: altos costos de los insumos y servicios, pues el $75.68 \%$ de las empresas lo manifestaron, y es también el principal problema para el caso del estado de Yucatán ya que el $70.88 \%$ de las unidades agropecuarias lo mencionaron (Tabla 2). También interfiere mucho en el avance de las unidades de producción la falta de capacitación y asistencia técnica, así como los precios bajos de los productos que comercializan. 


\section{Tabla 2}

Principales problemas a las que se enfrentan las empresas agropecuarias

\begin{tabular}{lcc}
\hline \multicolumn{1}{c}{$\begin{array}{c}\text { Problemas que afectan las actividades } \\
\text { agropecuarias }\end{array}$} & México & Yucatán \\
\hline Altos costos de insumos y servicios & 75.68 & 70.88 \\
Falta de capacitación y asistencia técnica & 33.15 & 22.16 \\
Pérdida de fertilidad del suelo & 28.44 & 18.99 \\
Infraestructura insuficiente para la producción & 24.03 & 28.4 \\
Excesivo intermediarismo & 22.77 & 20.74 \\
Precios bajos & 31.73 & 27.25 \\
Vejez, Enfermedad o invalidez del productor & 18.91 & 21.22 \\
Inseguridad & 16.99 & 10.65 \\
\hline
\end{tabular}

Fuente: Elaboración propia con base a los datos de la Encuesta Nacional Agropecuaria 2017.

En el caso Yucatán del total de las unidades agropecuarias el 6.27\% de ellas manifestaron que cuentan con maquinaria propia para realizar sus actividades, de ese total el $21.17 \%$ cuenta con tractor, el $6.42 \%$ con trilladoras, el $0.42 \%$ con motogrúas, sólo el $7.86 \%$ de esas unidades dijeron poseer sembradoras de precisión, mientras que el $84.24 \%$ manifestó que cuenta con otra maquinaria de uso agrícola. Evidentemente esto se encuentra orientado en mayor medida para las unidades de producción dedicadas a las actividades agrícolas (Encuesta Nacional Agropecuaria 2017).

Es fundamental tener claro el concepto de actividad agropecuaria, para lo cual, de acuerdo a la Real Academia Española (2016), citado por García (2017) "es todo lo que tiene relación con la agricultura y la ganadería"; es decir, es el uso y aprovechamiento de los recursos naturales renovables para la: agricultura, ganadería, silvicultura y acuacultura, son actividades que se pueden desarrollar dentro de un área específica, con la finalidad de otorgar un bienestar a las personas que se dedican a este tipo de actividad.

Del mismo modo Guerra, citado por García (2017) considera que los responsables de la administración de empresas agrícolas requieren "estar directamente relacionados con el proceso de producción". En este sentido es importante realizar la planificación, ejecución y control de las actividades en torno a la producción, es decir es necesario considerar los insumos 
DIAGNÓSTICO DE LA SITUACIÓN DE LAS MIPYMES DEL SECTOR AGROPECUARIO EN YUCATÁN.

que se requieren, los tiempos en las que se llevarán a cabo, así como la mano de obra que se empleará.

Por otro lado, de acuerdo con la Organización de las Naciones Unidas para la Alimentación y la Agricultura (1985) citado por Troncoso (2011) define la empresa agrícola como "conjunto de recursos asociados al recurso tierra, bajo una misma administración cuyo objetivo es la producción agrícola, ganadera, forestal y piscícola". Es decir, es un conjunto integral en donde el elemento principal es el recurso natural como el todo que permitirá con la aplicación de las herramientas de la administración su rentabilidad y alcanzar las metas propuestas. Del mismo modo para Aguilar (1989) citado por Márquez (2002) "la administración de empresas agropecuarias es el proceso de planeación y organización que lleva consigo la responsabilidad de integrar, dirigir y controlar en forma eficiente las actividades"; es decir, en esta definición se hace presente de manera directa el proceso de la administración con el fin de llevarlo de manera exitosa para que la empresa alcance la meta propuesta y fijado en la etapa de planeación.

Es importante para las empresas agropecuarias como en toda organización, tener definido su estructura organizativa con la cuál puedan funcionar de manera efectiva y eficiente, esto con la finalidad de tener claridad en la ejecución de las actividades, para esto existen varias teorías que establecen principios sobre cómo debería estar organizada la empresa, por ejemplo la teoría burocrática de Max Weber citado por Guerra (1998) que plantea que en la empresa debe tener: a) una clara división de trabajo, b) cada jefe tiene claramente definida su relación con otros jefes y subordinados de acuerdo con una jerarquía formal, c) existe confianza en políticas, reglas específicas y procedimientos que guían la conducta personal, d) El favoritismo es minimizado por medio de la aplicación impersonal de políticas, reglas y castigos, e) Se utilizan criterios rígidos y equitativos par la selección de candidatos. Esto claramente indica una estructura jerárquica lineal que va del director a los jefes de áreas, por tanto esta estructura organizacional es la que más se ha utilizado y aun hoy en día se sigue utilizando tanto en las empresas privadas como públicas, sin embargo, existen otras teorías que consideran la condición de los trabajadores para poder desarrollar e incrementar la productividad en la empresa como por ejemplo la teoría X y Y de Mcgregor (1960), en donde la estructura de la organización debería apostar por sacar de cada empleado lo mejor de sí, y no pensar en una estructura total mente rígida. 
DIAGNÓSTICO DE LA SITUACIÓN DE LAS MIPYMES DEL SECTOR AGROPECUARIO EN YUCATÁN.

Es así, como los elementos de la ciencia administrativa se presentan en las empresas agropecuarias, las cuales son Planificar, Organizar, Dirigir y Controlar, que son los elementos necesarios para orientar a la empresa a lograr sus metas.

De acuerdo con Chiavenato (2006) "la administración es el proceso de planear, organizar, dirigir y controlar el uso de los recursos para lograr los objetivos organizacionales". Ante esto, en toda empresa es importante seguir al pie el ciclo administrativo para alcanzar los resultados que se propongan, quizás en las empresas agropecuarias por su naturaleza su aplicación debiese ser mucho más puntual, porque la actividad esta influencia por fenómenos climáticos y biológicos que pueden perjudicar los resultados planteados.

Entonces, en toda empresa ya sea del sector industrial, de servicios o agropecuaria para su correcta administración debe seguir de manera continua la aplicación del proceso administrativo, ya que con cada etapa le permite al tomador de decisión conocer los requerimientos necesarios para conseguir los resultados planteados. Por ejemplo, en la etapa de planificación se debería responder el, ¿qué producir? ¿cuánto producir?, ¿cómo producir?, una vez resuelta estas interrogantes, es menester, que en la etapa de la organización se defina la estructura de la empresa, el flujo de la información y la estructura de autoridad que se empleará en la organización, así como el tipo de empresas agropecuaria que será, el apartado de ejecución se refiere a poner en marcha lo ya planificado con el fin de alcanzar las metas trazadas, y en el control es necesario diseñar indicares que permitan medir la rentabilidad y productividad de la empresa.

Sin embargo, todo esto debe ser un proceso continuo, al finalizar cada etapa se inicia un nuevo proceso con el fin de detectar lo que funciona o no, para corregir en la gestión ideal de la empresa agropecuaria, deben de existir tres grandes áreas las cuales son: Producción, Marketing (mercadeo) y finanzas.

El objetivo del presente trabajo es identificar la situación administrativa que predomina en las microempresas agropecuarias en nueve municipios del Estado de Yucatán, que permita determinar los principales problemas que se presentan en su administración y organización lo cual impiden su crecimiento y consolidación en el mercado.

\section{MATERIAL Y MÉTODO}


El estudio es de enfoque cuantitativo, de alcance descriptivo y de corte transversal, debido a que se realizó una encuesta a los productores en un solo momento. El instrumento contenía secciones como: forma en la que se administra el negocio; es decir, quién o quiénes toman la decisión, tipo de comunicación establecida en la empresa, forma de controlar las ventas y lo referente al área de finanzas.

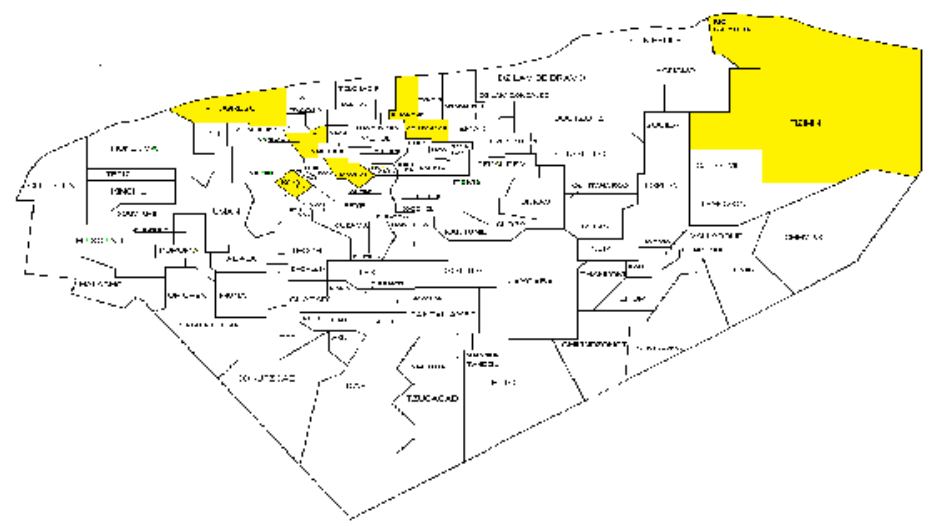

Figura 1. Ubicación de las empresas de estudio.

Las empresas se eligieron a través del muestreo no aleatorio y por conveniencia, esto debido a que se tuvo contacto de manera previa con los dueños y encargados de las empresas ubicados en los diferentes municipios, esto con la finalidad de tener una distribución geográfica de las mismas en el estado, tal como se observa en la (Figura 1), a cuyos encargados (dueños o directores generales) se le aplicó el cuestionario que constó de 24 preguntas, dividido en cuatro apartados. Los criterios seguidos para la aplicación del cuestionario fueron: A todos los encargados se les aplicó el mismo instrumento en un mismo periodo de tiempo, de manera libre y sin presiones para que contestaran el instrumento, se realizó en un ambiente adecuado, lo cuál les permitió a los informantes expresar libremente sus ideas respecto del problema.

En el primer apartado del instrumento se refiere a la identificación de la empresa, pues incluye preguntas, como: ¿Dónde se ubica la empresa?, año de inicio de operaciones, número de empleados que tiene, entre otros; en la sección 2, se refiere a la administración propia de la empresa, en la cual se trató de indagar sobre, la conformación de su negocio, ¿Quién toma la decisión?, estructura orgánica, canales de comunicación que se emplea, mecanismos de motivación para los empleados, mecanismos para establecer los salarios, factores que limitan su crecimiento; en la sección 3 correspondiente a Ventas, se preguntó por registro de ventas, estrategia de seguimiento de clientes, plan de mercadeo, así como conocer los factores que 
DIAGNÓSTICO DE LA SITUACIÓN DE LAS MIPYMES DEL SECTOR AGROPECUARIO EN YUCATÁN.

influyen en su proceso de venta. En el apartado IV Finanzas, se preguntó sobre los recursos para operar la empresa, si realiza un presupuesto por escrito, si conoce el margen de utilidad del producto, si realiza registro de operaciones contables y si tiene problemas de liquidez.

\section{RESULTADOS}

Las empresas entrevista del sector agropecuario se encuentran en los siguientes municipios del estado de Yucatán tal como se menciona en la Tabla 3, de igual manera se puede observar que la empresas agropecuarias entrevistadas, tienen diferentes años de antigüedad de inicio de operaciones tal como es el caso de la empresa ubicada en el municipio de Tixkokob que inició operaciones en el año 1990, seguida del de Progreso que inició en 1999, posterior a esa destacan las empresas ubicadas en los municipio de Mocochá y Sinanche que iniciaron operaciones en el año 2006 y 2007 respectivamente, la empresa de Río Lagartos inició operaciones en el 2012, mientras la ubicada en el municipio de Cansahcab se estableció en el 2015 y los de los municipios Conkal y Kanasín iniciaron sus actividades en el año 2016, en tanto que la empresa de Tizimin empezó su actividad en el 2017.

\section{Tabla 3}

Localización de las empresas

\begin{tabular}{lc}
\hline \multicolumn{1}{c}{ Municipio } & Año de inicio/actividad \\
\hline Sinanche & 2007 \\
Progreso & 1999 \\
Conkal & 2016 \\
Kanasín & 2016 \\
Tizimin & 2017 \\
Mocochá & 2006 \\
Cansahcab & 2015 \\
Tixkokob & 1990 \\
Río Lagartos & 2012 \\
\hline
\end{tabular}

Fuente: Elaboración propia con datos del cuestionario aplicado 2019.

De las nueve empresas entrevistas el 77\% son microempresas pues tienen de 1 hasta 10 empresas mientras que el $23 \%$ se clasificaron como pequeñas empresas esto debido a que tienen de 12 hasta 35 empleados. De estas empresas se tiene que el 34\% está dedicado a la actividad Avícola es decir a la cría de gallinas y producción de huevo, mientras que con el 22\% destaca la ganadería y la cría y engorda de cerdo, y el 11\% de estas empresas se dedica a la cunicultura y a la agricultura (Figura 2). 


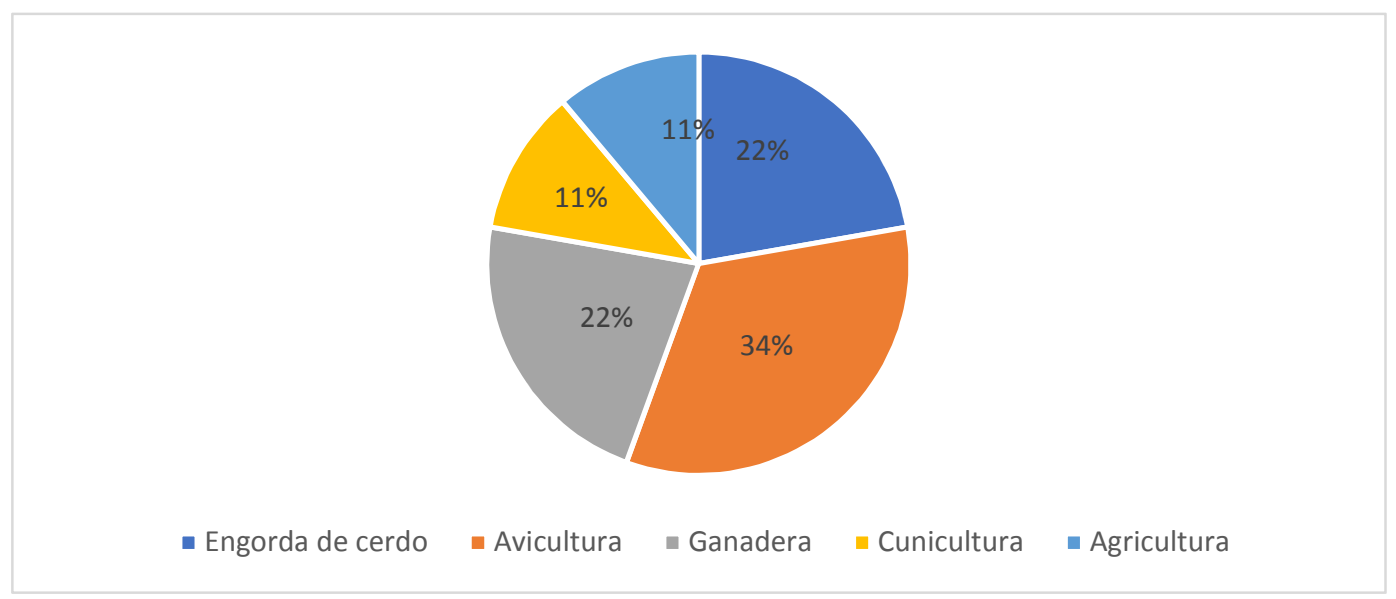

Figura 2. Tipo de actividad realizada por las empresas.

Fuente: Elaboración propia con base a los datos del cuestionario aplicado 2019.

Del mismo modo, en la forma de propiedad de estas empresas destaca que el $44 \%$ son de un solo dueño, igual porcentaje se obtuvo de los que mencionaron que son empresas familiares y sólo el $12 \%$ manifestó que la empresa estaba constituida como un corporativo.

En cuanto a la forma de su organización administrativa destaca que el $77.7 \%$ de las empresas encuestados manifestaron que tienen bien establecido las actividades que deben desarrollar los trabajadores, y solamente el $22.3 \%$ de ellas manifestaron que los trabajadores tienen diversas actividades por realizar dentro de la empresa. El 55.5\% de estas empresas entrevistadas manifestaron tener una pequeña estructura orgánica; sin embargo, no la siguen tal cuál como la tienen, pues no han generados manuales de organización para realizar y hacer más eficiente las actividades en la empresa, mientras que el $44.5 \%$ dijo no tener una estructura orgánica definida.

En cuanto a los medios que tienen para comunicar las tareas dentro de la empresa, todas mencionaron que utilizan la comunicación directa, es decir de persona a persona, así como también emplean con frecuencia el teléfono y la mensajería para dar a conocer alguna información importante a sus trabajadores. Al preguntarle a los gerentes sobre qué tan eficiente consideran la toma de decisiones dentro de la empresa, se tuvo que en el $33 \%$ de los casos considera que se toman de manera eficiente las decisiones, en tanto que el $55 \%$ considera que las decisiones son tomadas de manera deficiente y el $12 \%$ considera la toma de decisiones en la empresa como regular. En cuanto a la comunicación que se da en la empresa, el 55\% de los entrevistados mencionaron que es eficiente entre los empleados y la gerencia. 
DIAGNÓSTICO DE LA SITUACIÓN DE LAS MIPYMES DEL SECTOR AGROPECUARIO EN YUCATÁN.

En las empresas se mencionaron varias maneras de motivar a los empleados, destacando los siguientes: estímulos económicos, días de descanso adicional, bonos, reconocimientos entre otros, de igual manera en todas las empresas donde se aplicó la entrevista los directivos coincidieron que es de suma importancia que los empleados, directivos o dueño, así como clientes estén motivados, ya que eso permite que la empresa alcance sus objetivos que se ha planteado.

Algunas de las dificultades que presentan las empresas y que se pueden vincular a la deficiencia en su estructura organizativa y administrativa (Figura 3), de igual manera, como no tienen un área en particular establecida para el manejo de los recursos y el análisis financiero de la misma, esto los lleva a no tener un control riguroso sobre los activos de la empresa, lo cual se refleja en problema de la liquidez para hacer frente a sus obligaciones de manera rápida, y en problemas para obtener financiamiento, aunado también a su condición de microempresa. Pero también es de igual importancia el conflicto que se presenta con el personal y la falta de los espacios adecuados en las áreas para el trabajo, todo esto vinculado a una mala organización y manejo administrativo.

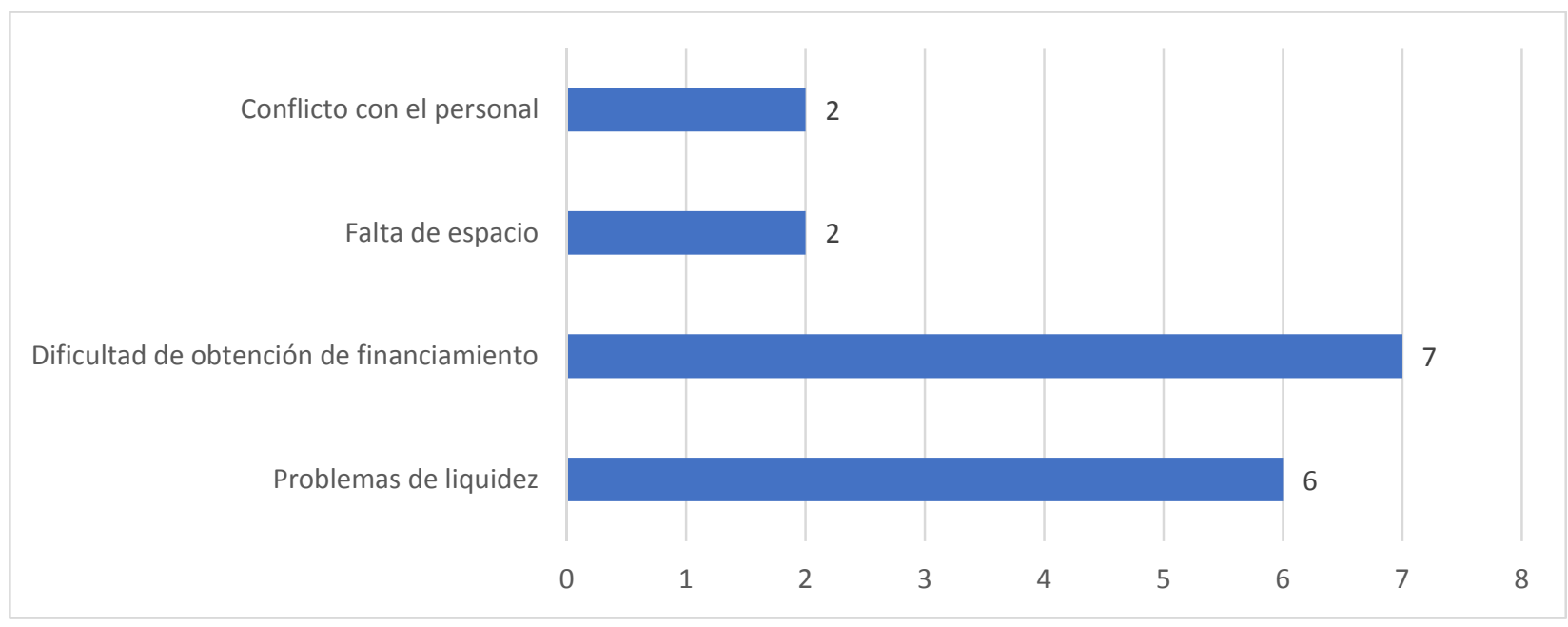

Figura 3. Principales inconvenientes que presentan las empresas.

Fuente: Elaboración propia con resultados del cuestionario aplicado 2019.

De las expectativas que tienen para el crecimiento de su empresa en el mediano plazo, el 100\% manifestó que buscarán el crecimiento de sus empresas mediante el incremento en las ventas, el $77.7 \%$ buscará incrementar sus ventas mediante de nuevos clientes locales y el $66.6 \%$ manifestó que lo hará mediante clientes fuera del municipio y del estado. 
DIAGNÓSTICO DE LA SITUACIÓN DE LAS MIPYMES DEL SECTOR AGROPECUARIO EN YUCATÁN.

De las variables que pueden afectar a las empresas para una posible inversión en el mediano plazo destacan: búsqueda de nuevos clientes, innovación en los procesos, remodelación y ampliación, mejoramiento del recurso humano y que existan política de gobierno.

En cuanto a los factores que podrían limitar el crecimiento de la empresa, destaca la falta de recursos para invertir, ya que del total de los responsables de las empresas que fueron entrevistados, siete de ellos contestaron entre muy importante e importante, para ellos esa variable, así como la disponibilidad de la materia prima o los recursos para realizar el proceso de producción, ya que sin estos elementos se estancaría la empresa. En menor medida destaca la dificultad para conseguir personal adecuado para las actividades del sector agropecuario.

Como puede apreciarse en la figura 4, en la sección de ventas, que de las nueve empresas entrevistadas, siete de ellas si llevan a cabo un registro diario de ventas, y dos de ellas no las realiza. En cuanto al análisis de la competencia, en cinco empresas se realizan pequeños estudios de mercado para conocer el comportamiento, y cuatro de ellas no las considera importante; en la variable inversión en mercadotecnia, solo cuatro empresas la realizan para promocionar su producto, y los restantes consideran que su producto ya está posicionado y no requiere más inversión en publicidad; por último, un elemento importante que ayuda a darle control a las actividades dentro de la empresa es, el manual de procedimientos; sin embargo, esta no se tiene en siete de las empresas entrevistadas, sólo en dos si lo consideran

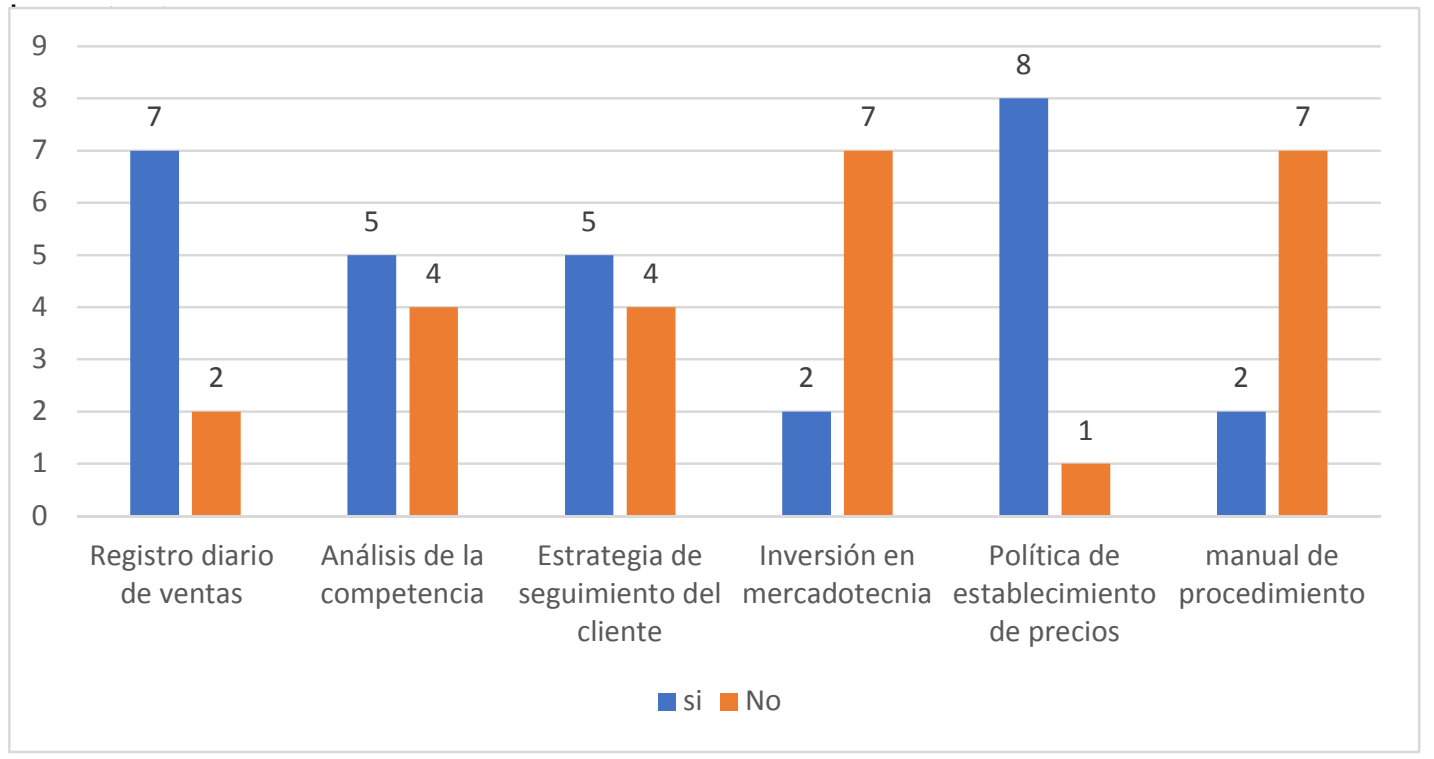

Figura 4. Variables para el control de ventas.

Fuente: Elaboración propia con datos del cuestionario aplicado 2019.

MOO-NOVELO, C. A., HERNÁNDEZ-CUEVAS, F. I., MEX-ARRIAGA, F. I. 
En el caso particular de las variables que pudieran afectar el proceso de ventas en las empresas, destaca en primer lugar la calidad del producto, como aquel que consideran de mayor relevancia las empresas, le sigue con el 55.5\% de importancia la variable diferenciación del producto con respecto a la competencia, aquí más que la diferenciación directa, se refiere al valor agregado que cada empresa le da a su producto final, para satisfacer las necesidades del cliente.

\section{DISCUSIÓN}

Al contrastar estos resultados que se obtuvieron de las empresas con los de la Encuesta Nacional Agropecuaria del 2017, se puede observar que existen similitudes: las empresas siguen siendo de tamaño micro, y pequeñas en cuya importancia radican que son las que más generan empleos para la sociedad en su conjunto. En estas empresas no se aplica de manera directa el proceso de administración, pues en la parte de la organización estas empresas carecen de una estructura orgánica bien estructurada, así como una carencia en la realización de un seguimiento puntual en el registro de sus operaciones ya que no la realizan de manera formal en alguna base de datos, en la mayoría de estas empresas el dueño es el que toma las decisiones no solo de producción sino también mercadeo del producto, así como también de finanzas. Tampoco adoptan un análisis de mercado riguroso que les permita reaccionar ante los cambios en los gustos y preferencias de los consumidores.

Del mismo modo no se identifica de manera directa las tres áreas fundamentales que de acuerdo con Guerra (1998) son importantes para una correcta administración y toma de decisiones en las empresas agropecuarias las cuales son: producción, mercadeo y finanzas, estas áreas como tal no se distinguen en la estructura de estas empresa analizadas, esto debido a que precisamente como se indicó carecen de una adecuada estructura organizacional, que les permita identificar las funciones específicas de cada área, con lo que podrían reducir tiempos en las actividades y facilitaría el análisis de la situación que se presenta en las empresas, esta deficiencia en la estructura organizacional de estas pequeñas empresas precisamente les impiden consolidarse y crecer, y por tanto, también no pueden acceder a créditos, y tampoco generar procesos de integración en cadenas de suministros.

La carencia de la aplicación de la planeación estratégica en las empresas trae como consecuencia impedimentos al momento de establecer su filosofía empresarial y sus objetivos que pretendan alcanzar, la mayoría de las empresas llevan una administración empírica. 
DIAGNÓSTICO DE LA SITUACIÓN DE LAS MIPYMES DEL SECTOR AGROPECUARIO EN YUCATÁN.

Dos de las problemáticas identificadas con este estudio es en primer término la escasez de la materia prima y el encarecimiento de estas, estas son a juicio de los entrevistados los elementos que repercuten en el crecimiento de estas empresas, pues sus costos se incrementan, con lo que ven reducidos sus ingresos y por tanto se reduce la reinversión. También se identificó la falta de acceso a financiamiento, esto ocurre por su mala organización administrativa lo cual no les permite generar sus reportes y cálculo de indicadores que evidencien la salud financiera de la empresa.

\section{CONCLUSIONES}

Es necesario que las empresas en el sector agropecuario tengan bien definida su estructura, para que apliquen de manera correcta el proceso administrativo. Otra de las conclusiones importantes a destacar es que las empresas se enfrentan a dificultades de acceso a los préstamos o créditos, así como a la falta de liquidez, todo esto por una falta de objetivos y metas bien establecidos, así como a su poco control del mercado y monitoreo del comportamiento del consumo de los clientes.

Otro elemento importante es la falta de inversión en mercadotecnia para la promoción de sus productos, ya que no la consideran necesaria, del mismo modo no cuentan con su manual de procedimiento, ni de organización por lo que muchos de los empleados no saben cómo realizar la actividad o cometen muchos errores en sus áreas de trabajo, lo cual reduce la productividad en estas empresas, esto aunado a la falta de capacitación, pues como son empresas micro, no consideran relevante estos aspectos. Un elemento para destacar es que el $55 \%$ de las empresas expresaron que tienen mecanismo para dar un seguimiento en cuanto a la satisfacción del cliente, así como el $88 \%$ de estas empresas manifestaron tener una política de fijación de precios para sus productos. En tanto que, uno de los principales problemas a las que se enfrentan estas empresas es a la falta de liquidez, pues esta problemática se manifestó en el $66 \%$ de los casos entrevistados.

\section{RECOMENDACIONES}

Existe una gran brecha que puede ser cubierta por las autoridades estatales y federales a través del diseño de políticas públicas acordes con la problemática que presenta este tipo de empresas, adicional que es importante que se dé un seguimiento puntual de la aplicación de 
DIAGNÓSTICO DE LA SITUACIÓN DE LAS MIPYMES DEL SECTOR AGROPECUARIO EN YUCATÁN.

programas orientados hacia el sector agropecuario, para saber si en verdad llega los recursos a quienes más lo requieren.

En el ámbito agropecuario se deberá buscar que las empresas realmente generen valor agregado a su producción y no solo sean vendedores de materias primas, para lo cual se requiere de la tecnificación de las empresas y que se ofrezcan créditos blandos para que estos pequeños productores puedan capitalizarse y así incrementar su productividad.

Por otra parte, la capacitación hacia la profesionalización de las empresas es vital, por lo que ofrecer espacios de aprendizaje donde se impartan cursos de capacitación, que sean acordes con las necesidades de este tipo de empresas por parte de las instituciones como la secretaría de desarrollo rural Federal, o través de la secretaría de desarrollo rural estatal, brindando talleres en temas de: administración, finanzas, marketing, adicional a las que se les da en áreas específicas de agricultura y ganadería.

\section{REFERENCIAS BIBLIOGRÁFICAS}

Centro de Estudios para el Desarrollo Rural Sustentable y la Soberanía Alimentaria. (2019). Reporte: Resultados de la Encuesta Nacional Agropecuaria 2017, Cámara de Diputados, LXIV legislatura, Palacio Legislativo de San Lázaro, Ciudad de México.

Chiavenato, I. (2007). Introducción a la Teoría General de la Administración (7ª̣ ed.). México: Mc Graw-Hill.

Encuesta Nacional sobre Productividad y Competitividad de las Micro, Pequeñas y Medianas Empresas (ENAPROCE). (2019). Consultado el 5 de mayo de 2019 https://www.inegi.org.mx/programas/enaproce/2015/default.html\#Tabulados

García Pérez, S. A. (2017). Las empresas agropecuarias y la Administración Financiera, Revista Mexicana de Agronegocios, vol. XXI, núm. 40, 2017, (583-594) Sociedad Mexicana de Administración Agropecuaria A.C. Universidad Autónoma del Estado de México, México Recuperado http://www.redalyc.org/jatsRepo/141/14152127007/html/index.html\#redalyc_14152127007 ref24

Góngora Pérez, J. P. (2013). El panorama de las micro, pequeñas y medianas empresas en México. Revista de Comercio Exterior, Vol, 63, No. 6 Nov-Dic. 2013. 
DIAGNÓSTICO DE LA SITUACIÓN DE LAS MIPYMES DEL SECTOR AGROPECUARIO EN YUCATÁN.

Guerra, G. (1998). Manual de Administración de empresas agropecuarias (2a ed.). Instituto Interamericano de Cooperación para la Agricultura, San José Costa Rica.

INEGI. (2019). Resultados de la Encuesta Nacional Agropecuaria (2017). Consultado 5 de julio de https://www.inegi.org.mx/programas/ena/2017/default.html\#Tabulados

INEGI. (2019). Resultados del Censo Económico (2019) consultado el 10 agosto en https://www.inegi.org.mx/programas/ce/2019/default.html\#Tabulados

Madero Gómez, S. M. y Rodríguez Delgado, D. R. (2018) Relación entre las Teorías X y Y de McGregor, las formas de retribuir y la satisfacción de las personas en su trabajo. Ciencias UAT, Universidad Autónoma de Tamaulipas, Ciudad Victoria, Jul-Dic. 2018, Vol 13 No.1

Marco, F. (2016). Introducción a la Gestión y Administración en las Organizaciones (2a ed.). Universidad Nacional Arturo Jouretche.

Márquez, M. (2002). La gestión Administrativa de las empresas agropecuarias de los municipios San Fernando y Biruaca del Estado Apure, en Venezuela, Revista Mexicana de Agronegocios, enero-Julio, Vol. 10 Sociedad Mexicana de Administración Agropecuaria A.C, La Universidad Autónoma de la Laguna, La Universidad Autónoma Agraria "Antonio Narro", Unidad Laguna Torreón, México, pp. 324-335.

Troncoso, J. L (2011). Principios de Administración de empresas agrícolas, Universidad de Tala. $\begin{array}{lllllll}\text { Consultado el } & 18 & \text { de } & \text { junio } & \text { de } & 2020 & \text { en }\end{array}$ file://C:/Users/Carlos\%20M/Downloads/Librodeadministracindeempresasagrcolas.pdf 
DIAGNÓSTICO DE LA SITUACIÓN DE LAS MIPYMES DEL SECTOR AGROPECUARIO EN YUCATÁN.

MOO-NOVELO, C. A., HERNÁNDEZ-CUEVAS, F. I., MEX-ARRIAGA, F. I. 